\title{
ASMA \\ Eventos serios en asma con \\ fluticasona/salmeterol en comparación con fluticasona: Estudio AUSTRI
}

\section{ASTHMA. Serious events in asthma with fluticasone/salmeterol in comparison with fluticasone: AUSTRI trial}

IVÁN Solarte Rodríguez, MD.(1); MANUela ToBón TRUJILLO, MD.(2)

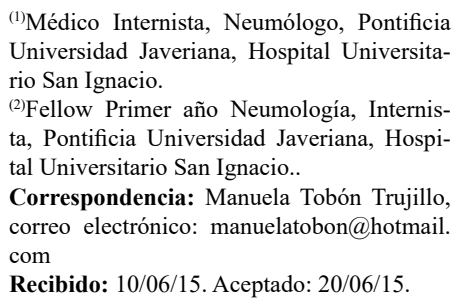

${ }^{(1)}$ Médico Internista, Neumólogo, Pontificia Universidad Javeriana, Hospital Universitario San Ignacio.

${ }^{(2)}$ Fellow Primer año Neumología, Internista, Pontificia Universidad Javeriana, Hospital Universitario San Ignacio..

Correspondencia: Manuela Tobón Trujillo, correo electrónico: manuelatobon@hotmail. com

Recibido: 10/06/15. Aceptado: 20/06/15.

\section{Referencia}

Stempel DA, Raphiou IH, Kral $\mathrm{KM}$, Yeakey AM, Emmett AH, Prazma CM, et al. Serious asthma events with fluticasone plus salmeterol versus fluticasone alone. N Engl J Med. 2016 Mar 6; [Epub ahead of print]

\section{Pregunta}

En pacientes con asma, ¿cuál es el riesgo de desenlaces adversos al administrar una combinación de corticosteroide inhalado (CI) y beta-agonista de larga acción (BALA) (fluticasona/salmeterol) en comparación con $\mathrm{CI}$ solo (fluticasona)?

\section{Diseño}

Estudio multicéntrico, de asignación aleatoria, doble ciego.

\section{Cegamiento}

Pacientes y clínicos evaluadores. El ciego del tratamiento se mantuvo respecto a los grupos fluticasona/salmeterol versus fluticasona, pero no en relación con la dosis del esteroide inhalado.

\section{Período de seguimiento}

Tratamiento activo 26 semanas, una semana de seguimiento posterior.

\section{Lugar}

710 centros en 33 países. Centros en Colombia: Bogotá: Hospital Universitario San Ignacio (Doctor Iván Solarte); Bucaramanga: Instituto Neumológico del Oriente (Doctor Fabio Bolívar).

\section{Pacientes}

Mayores de 12 años con asma moderada a severa, con historia de la enfermedad de al menos un año, con requerimiento de medicación diaria para lograr control y que hubieran recibido tratamiento con glucocorticoides sistémicos para el manejo de una exacerbación o hubieran requerido manejo intrahospitalario por una exacerbación de asma en los 12 meses previos, con exclusión de los 30 días previos a la aleatorización.

Los pacientes fueron excluidos si presentaban historia de asma que amenazara la vida, tabaquismo de más de 10 paquetes/año o asma inestable. 


\section{Intervención}

- Combinación de propionato de fluticasona más salmeterol en dosis de $100 \mathrm{mcg}$ de fluticasona y $50 \mathrm{mcg}$ de salmeterol, $250 \mathrm{mcg} / 50 \mathrm{mcg}$ y 500 $\mathrm{mcg} / 50 \mathrm{mcg}$, respectivamente.

- Propionato de fluticasona sola, en dosis de 100 mcg, $250 \mathrm{mcg}$ o $500 \mathrm{mcg}$, administrado dos veces al día en un inhalador de polvo-seco.

\section{Resultados}

Se incluyeron 11.679 pacientes, de los cuales 67 presentaron 74 eventos severos relacionados con asma, con 36 eventos en 34 sujetos en el grupo de fluticasona/salmeterol y 38 eventos en 33 pacientes en el grupo de fluticasona; HR de 1,03 (IC 95\% 0,64-1,66) .

Se reportaron una o más hospitalizaciones en 34 pacientes en el grupo de fluticasona/salmeterol y en 33 pacientes del otro grupo, para un total de 36 hospitalizaciones para cada uno de ellos (tabla 1).

El riesgo de exacerbaciones severas fue 21\% menor en el grupo de fluticasona/salmeterol que en el grupo de fluticasona sola (HR 0,79; IC95\% $0,70-0,89 ; \mathrm{p}<0,001)$, con al menos una exacerbación severa ocurrida en 480 de 5.834 pacientes en el grupo de fluticasona/salmeterol comparado con 597 en 5.845 sujetos en el grupo de fluticasona $(\mathrm{p}=0,001)$ (tabla 2).

En el análisis de subgrupos, el riesgo de exacerbaciones severas fue consistentemente menor en el grupo de fluticasona/salmeterol que en aquellos tratados con fluticasona sola, con un $35 \%$ menos de riesgo en el grupo de los adolescentes.

\section{Conclusiones}

Los pacientes que recibieron terapia con fluticasona/salmeterol no tienen riesgo mayor de presentar eventos serios relacionados con asma en comparación con aquellos que recibieron fluticasona sola. Los pacientes del grupo fluticasona/salmeterol tuvieron menos exacerbaciones severas que los del grupo de fluticasona sola.

\section{Comentario}

Hace mas de 30 años se relacionó el uso de terapia con broncodilatadores beta 2 agonistas de acción corta en asma con el incremento de eventos adversos severos y mortalidad $(1,2)$. Estas consideraciones se extendieron al uso de BALA, con los resultados evidenciados en estudios como el SNS (del inglés Serevent Nation-wide Surveillance) y SMART (del inglés Salmeterol Multicenter Asthma Research Trial) (3-6).

El uso de BALA ha demostrado tener un efecto favorable en cuanto a la disminución de la exacerbaciones, pero los estudios previamente mencionados no tenían un control estricto de la dosis y el uso concomitante de esteroide inhalado, de tal modo que ello podría explicar las exacerbaciones severas. Se había solicitado la realización de estudios con BALA y dosis controladas de esteroide inhalado para evaluar

Tabla 1. Desenlaces primarios de seguridad.

\begin{tabular}{|lcc|}
\hline \multicolumn{1}{|c|}{ Desenlace primario } & $\begin{array}{c}\text { Fluticasona/salmeterol } \\
\mathbf{n = 5 . 8 3 4}\end{array}$ & $\begin{array}{c}\text { Fluticasona } \\
\mathbf{n = 5 . 8 4 5}\end{array}$ \\
\hline Desenlace compuesto $\mathrm{n}(\%)$ & $33(<1 \%)$ & $33(<1 \%)$ \\
- Muertes relacionadas con asma & 0 & 0 \\
- Intubación relacionada con asma & 9 & $33(<1 \%)$ \\
- Hospitalización relacionada con asma & $34(<1 \%)$ & 36 \\
Número total de hospitalizaciones relacionadas con asma & 36 & $6(<1 \%)$ \\
Muerte de cualquier causa n y \% & $3(<1 \%)$ & 2 \\
\hline
\end{tabular}


Tabla 2. Análisis de subgrupos: primera exacerbación severa.

\begin{tabular}{|c|c|c|c|c|}
\hline Subgrupo & $\begin{array}{l}\text { Fluticasonal } \\
\text { salmeterol }\end{array}$ & Fluticasona sola & $\begin{array}{l}\mathrm{HR} \\
\text { IC } 95 \%\end{array}$ & Valor $\mathbf{P}$ \\
\hline \multicolumn{5}{|l|}{ Control del asma } \\
\hline $\begin{array}{l}\text { - Previamente sin control con } \mathrm{Cl} \\
\text { sin BALA }\end{array}$ & $91 / 1.405(6)$ & 106/1.398 (8) & $0,83(0,63-1,10)$ & 0,20 \\
\hline $\begin{array}{l}\text { - Previamente sin control con } \mathrm{Cl} \\
\text { más BALA }\end{array}$ & $102 / 1.016(10)$ & $124 / 1.040(12)$ & $0,84(0,65-1,09)$ & 0,19 \\
\hline $\begin{array}{l}\text { - Previamente adecuado con } \mathrm{Cl} \\
\text { más BALA }\end{array}$ & $239 / 2.652(9)$ & $304 / 2.663(11)$ & $0,76(0,65-0,91)$ & 0,002 \\
\hline $\begin{array}{l}\text { - Previamente buen control con } \\
\mathrm{Cl}\end{array}$ & $38 / 612(6)$ & $54 / 608(9)$ & $0,68(0,45-1,03)$ & 0,07 \\
\hline \multicolumn{5}{|l|}{ Edad (años) } \\
\hline - $12-17$ & $42 / 615(7)$ & $64 / 615(10)$ & $0,65(0,44-0.95)$ & 0,03 \\
\hline - 18-64 años & $386 / 4.576(8)$ & $469 / 4.605(10)$ & $0,81(0,71-0,93)$ & 0,002 \\
\hline - Mayor de 64 años & $52 / 643(8)$ & $64 / 625(10)$ & $0,78(0,54-1,12)$ & 0,17 \\
\hline
\end{tabular}

el riesgo de añadir BALA al tratamiento con esteroide inhalado. La existencia de presentaciones combinadas de BALA y corticosteroide inhalado facilitó la realización de este estudio.

Con los resultados del estudio AUSTRI es posible concluir que en pacientes sin antecedentes de eventos que amenacen la vida relacionados con asma, como lo fueron los sujetos incluidos en este trabajo, el uso de la combinación fija fluticasona/salmeterol muestra un perfil de seguridad similar al de fluticasona sola y está asociado con un menor riesgo de exacerbaciones.

Estas conclusiones no pueden ser extrapolables a aquellos pacientes con historia previa de eventos serios asociados a asma o con exacerbaciones en los últimos 30 días, grupo con mayor probabilidad de presentar eventos serios relacionados con asma durante un periodo similar al de seguimiento del estudio, de ahí que el perfil de seguridad del uso de CI/BALA sigue siendo una pregunta sin resolver en aquel grupo de sujetos.

Adicionalmente, consideramos que la comparación de esteroide inhalado a la misma dosis en la combinación o en el esteroide solo, ofrece una ventaja frente a la combinación, en cuanto a la disminución de exacerbaciones que ya ha sido demostrada en estudios previos.

\section{Bibliografía}

1. Ernst P, Habbick B, Suissa S, et al. Is the association between inhaled beta agonist use and life-threatening asthma because of confounding by severity? Am Rev Respir Dis. 1993;148:75-9.

2 Spitzer WO, Suissa S, Ernst P, et al. The use of beta-agonist and the risk of death and near death from asthma. N Engl J Med. 1992;326:501-6.

3. Castle W, Fuller R, Hall J, Palmer J. Serevent nationwide surveillance study: comparison of salmeterol with salbutamol in asthmatic patients who require regular bornchodilator treatment. BMJ 1993;306:1034-7.

4. Nelson HS, Weiss ST, Bleecker ER, Yancey SW, Dorinsky PM. The Salmeterol Multicenter Asthma research Trial: a comparison of usual pharmacotherapy for asthma or usual pharmacotherapy plus salmeterol. Chest. 2006;129:15-26.

5. Weatherall M, Wijesinghe M, Perrin K, Harwood M, Beasley R. Meta-analysis of the risk of mortality with salmeterol and the effect of concomitant inhaled corticosteroid therapy. Thorax 2010;65:39-43.

6. Salpeter SR. An update on the safety of long-acting beta agonists in asthma patients using inhaled corticosteroids. Exper Opin Drug Saf. 2010;9:407-19. 